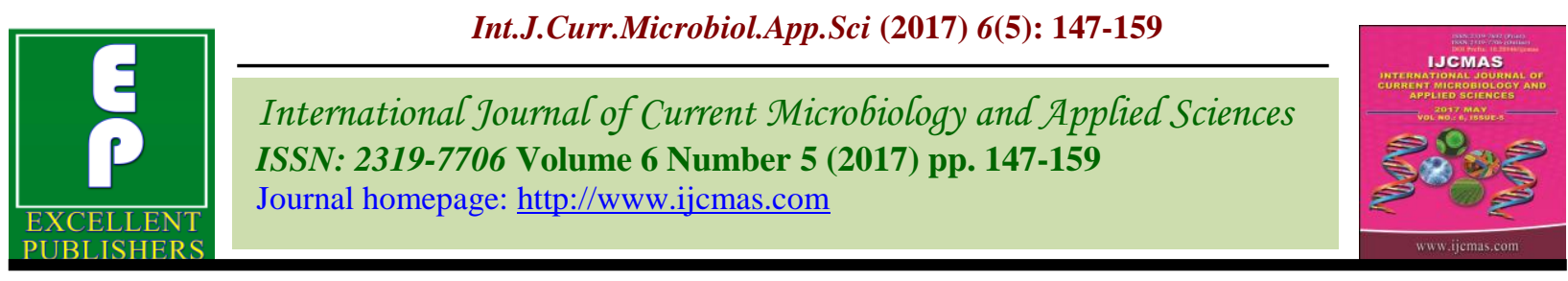

Original Research Article

https://doi.org/10.20546/ijcmas.2017.605.018

\title{
Effect of Moisture Stress on Key Physiological Parameters in Sunflower Genotypes
}

\author{
A. Geetha*, A. Sivasankar, P. Saidaiah and Lakshmi Prayaga \\ Department of Plant Physiology, College of Agriculture, ANGRAU, Rajendranagar, \\ Hyderabad (A.P.) - 500 030, India \\ *Corresponding author
}

\begin{tabular}{|c|c|}
\hline & A B S T R A C T \\
\hline Keywords & \multirow{4}{*}{$\begin{array}{l}\text { A study was conducted to investigate the effect of drought on physiological characteristics } \\
\text { in twelve sunflower genotypes. Moisture stress treatment were imposed at flower bud } \\
\text { initiation stage (irrigation withheld from } 40 \text { DAS to } 60 \text { DAS) whereas, Control plots were } \\
\text { irrigated at } 10 \text { days intervals throughout the crop growth period. Results revealed that } \\
\text { water stress showed repressing effect on Relative water content, Photosynthetic rate, leaf } \\
\text { fluorescence, membrane leakage, chlorophyll content and specific leaf area in all the } \\
\text { genotypes examined. Decline in Specific leaf area under water stress is considered as } \\
\text { adaptation to water stress. However, genotypic variation was significant for characters } \\
\text { studied. Genotypes SH-177, SH- } 491 \text { and DSF-111 was considered as promising lines by } \\
\text { maintaining higher RWC, photosynthetic rates, leaf fluorescence and SPAD chlorophyll } \\
\text { meter readings wither lower membrane leakage and Specific leaf area. }\end{array}$} \\
\hline $\begin{array}{l}\text { Drought, } \\
\text { Sunflower, RWC, } \\
\text { Photosynthetic } \\
\text { rate, } \\
\text { SPAD readings, }\end{array}$ & \\
\hline Article Info & \\
\hline $\begin{array}{l}\text { Accepted: } \\
\text { 04 April } 2017 \\
\text { Available Online: } \\
10 \text { May } 2017\end{array}$ & \\
\hline
\end{tabular}

\section{Introduction}

Sunflower (Helianthus annuus L.) occupies a prominent place among oilseed crops as it contributes about $12 \%$ to the world edible oil production. In India, sunflower is cultivated in an area of $21.6 \mathrm{~m}$ ha with an annual production $1.32 \mathrm{~m}$ tones. In fact, large area under sunflower is cultivated under rainfed situation, where intermittent moisture stress is most prevalent. The decrease in productivity in oilseeds in general and in sunflower in particularly is mainly due to abiotic stresses. Drought is the most limiting of all abiotic stresses as it causes more than $70 \%$ reduction in biomass and seed yield in sunflower (Umashaanker, 1991). Although sunflower has good potential to tolerate drought because of well developed root system. The productivity is still affected by drought. If drought tolerant sunflower hybrids/ varieties are developed, sunflower can be grown successfully under water limiting conditions.

Researchers have linked various physiological traits of plants to drought with their tolerance mechanisms. Among this, relative leaf water contents (RWC) is best measure to level the water deficit in the plant at a specific point of time. As RWC is related to cell volume, it may closely reflect the balance between water supply to the leaf and transpiration rate (Sinclair and Ludlow, 1985). The measurement of solute leakage from plant tissue is a long standing method for estimating membrane integrity in relation to 
environmental stresses, growth and development, and genotypic variation. In this regard, the degree of stability of cell membrane is considered to be one of the best physiological indicators of drought stress tolerance.

The use of chlorophyll fluorescence from intact attached leaves proved to be a reliable, non intrusive method for monitoring photosynthetic events and for judging the physiological status of the plant. Fluorescence induction patterns and derived indices have been used as empirical diagnostic tools in stress physiology. Photosynthesis response to water stress is poorly understood in sunflower. The extent to which photosynthetic capability is maintained during periods of water stress and the ability for rapid recovery of photosynthesis after re watering is important in crop adaptation to drought environments. Hence, Present investigation was carried out to study physiological traits like RWC, photosynthetic rate, Leaf fluorescence, membrane integrity, SPAD and SLA in twelve genotypes of sunflower under drought conditions.

\section{Materials and Methods}

The experiment was laid out in factorial Random Block Design with two factors and 12 treatments which were replicated thrice during rabi, 2010-11 at College Farm, College of Agriculture, ANGRAU, Rajendranagar, Hyderabad. Control (irrigated) and water stress was used as factors. Control plots were irrigated at 10 days intervals throughout the crop growth period whereas, in stress treatment irrigation withheld from 40 DAS to 60 DAS. The treatments comprised of 12 lines. Each genotype was sown in two rows at $5 \mathrm{~m}$ length with spacing of $60 \times 30$ $\mathrm{cm}$. Two to three seeds were sown per hill to achieve uniform stand. Thinning was done at two weeks after sowing to retain one seedling per hill. Recommended package of practices were followed to raise a healthy crop. In each entry, five plants were tagged randomly. Observations were recorded at 45,60 and 75 DAS i.e., during 5 days after imposition of stress, twenty days after imposition of stress and 15 days of stress recovery period on RWC, photosynthetic rate, Leaf fluorescence, membrane integrity, SPAD and SLA.

Leaf disc of approximately $4 \mathrm{~cm}^{2}$ area in rectangle shape was taken from plant under irrigated and stressed regimes and fresh weight was measured. Discs were then dipped in glass vials containing $20 \mathrm{ml}$ of deionized water. These veils were left for four hours at room temperature. After four hours, leaf discs were blotted and their turgid weight was recorded by formula as given below.

RWC $=($ Fresh weight-Dry weight $/$ Turgid weight -Dry weight) $X_{100}$

Photosynthetic rate is calculated using is portable infrared gas analyzer (IRGA). The optimal and effective quantum yields of PSII were measured using the fluorometer OS-500 (Opti-Science, USA). Membrane leakage was measured using automatic conductivity meter. Single leaf disks $1 \mathrm{~cm}$ diameter were excised with a leaf punch from the fourth main stem leaf, one disc per variety per treatment from similar interveinal areas were taken, and placed into trays with individual cells containing $2 \mathrm{ml}$ double de- ionized water. The electrical conductivity as a measure of cell leakage was read $48 \mathrm{hrs}$ after the leaf disks were placed in double de- ionized water at room temperature. The resulting electrical conductivity of the ion concentration in the solution depended on the leakage from the leaf disk. Chlorophyll concentration was assessed using a chlorophyll meter (SPAD502, Minolta).Measurements being taken at three points of each leaf (upper, middle and lower part).Average of these three readings was considered as SPAD reading of the leaf. Recording of SPAD readings was carried out 
fortnight intervals starting from 45DAS, in the $\left(3^{\text {rd, }} 5^{\text {th }}\right.$ and 7 th $)$ leaf to the top of the plant. The mean of SCMR reading was taken out in the end and presented as average SPAD value. Specific leaf area (SLA), an indication of leaf thickness was measured by taking the fully expanded leaf, more specifically the fifth leaf from the top, the leaf area was measured using leaf area meter. Later, the leaf was kept for drying at $80^{\circ} \mathrm{C}$ for 3-4days and once the leaf was dried, leaf weight was taken and SLA was computed as per the equation given below.

SLA $\left(\mathrm{cm}^{2} / \mathrm{g}\right)=$ Leaf area/ Leaf weight.

\section{Results and Discussion}

Relative water content (RWC) decreased with increase in stress duration. Relative water content was reduced from $(15 \%)$ at 45 DAS to $(26 \%)$ at 60 DAS compared to their controls (Table 1). At 15 days after release of stress, genotypes varied significantly in RWC content.SH-491 followed by $\mathrm{SH}-177$ under control and only SH-177 under stress recorded higher RWC \% over remaining genotypes, while, $\mathrm{SH}-177$ retained highest relative water content than rest of cultivars in interaction. Under water stress some genotype maintains its RWC at par with that of non stress conditions due to production of osmoprotectants or Compatible solutes and this compound reduces osmotic potential (Jha and Singh, 1997).

Photosynthetic rate was significantly reduced under stress conditions compared to control (Table 2). Percent reduction of photosynthetic rate was highest $(11.68 \%)$ at 75 DAS than at 60 DAS (10.68\%) and 45 DAS (10.52\%) compared to controls (Table 2). Among sunflower genotypes DSF-111 under control, whereas SH-177 followed by TSF-103 and RSF-106 under stress showed superior photosynthetic rates than other genotypes at 75 DAS. However, in interaction SH-177 showed highest photosynthetic rate followed by TSF -103, DSF-111, RSF-106 and RSF101 recorded on par and were significantly superior over other genotypes at recovery period (75 DAS). Decrease in photosynthetic rate is due to increase in stomatal resistance due to partial closure of stomata as well as difference in activation states of photosynthetic enzymes (Lawlor, 2002). The limitation of photosynthesis under drought through metabolic impairment is more complex phenomenon than stomatal limitation and mainly it is through reduced photosynthetic pigment contents in sunflower (Reddy et al., 2004).

Stress imposition at flower bud initiation stage resulted in significant difference in initial fluorescence between treatments throughout stress period. Maximum initial fluorescence was recorded at 5 days after initiation of stress (89.29) compared to control (96.14) (Table 3). At 15 days after release of stress, genotype DSF-104 recorded highest initial fluorescence in control, stress and interaction of genotype $\mathrm{x}$ treatments. Maximum fluorescence (Fo) was initially high at 5 days after imposition of stress, thereafter decrease was seen at 20 days after stress imposition and 15 days after release of stress. The maximum fluorescence differed significantly from 45 DAS onwards (Table 4). Among the stress treatments, the reduction in maximum fluorescence was more at 15 days after release of stress $(17.39 \%)$ than at remaining stages compared to their controls. Among genotypes, significant difference was found in maximum fluorescence from 45 DAS onwards. At recovery period (75 DAS), genotype ASF-107 under control and DSF114 followed by $\mathrm{SH}-177$ under stress exhibited high maximum fluorescence among genotypes. But in combined effect, $\mathrm{SH}-177$ recorded maximum (Fm) value followed by DSF-114. The decrease of Fv/Fm after severe water stress was recently reported by Miyashita et al., (2004). 
Table.1 Mean of relative water content (RWC) (\%) of sunflower cultivars during stress and after stress influenced by moisture stress

\begin{tabular}{|c|c|c|c|c|c|c|c|c|c|c|c|c|c|}
\hline \multirow[b]{2}{*}{ S.No. } & \multicolumn{5}{|c|}{ Five days after imposition of stress } & \multicolumn{4}{|c|}{$\begin{array}{c}\text { Twenty days after imposition of } \\
\text { stress }\end{array}$} & \multicolumn{4}{|c|}{ Fifteen days after release of stress } \\
\hline & Genotype & Control & Stress & Mean & $\begin{array}{c}\% \\
\text { decrease }\end{array}$ & Control & Stress & Mean & $\begin{array}{c}\% \\
\text { decrease }\end{array}$ & Control & Stress & Mean & $\begin{array}{c}\% \\
\text { decrease }\end{array}$ \\
\hline 1 & RSF-101 & 73.50 & 70.84 & 72.17 & 3.61 & 74.10 & 64.30 & 69.20 & 13.23 & 75.90 & 72.20 & 74.05 & 4.87 \\
\hline 2 & TSF-103 & 85.13 & 75.64 & 80.38 & 11.14 & 84.03 & 64.43 & 74.23 & 23.32 & 87.17 & 73.57 & 80.37 & 15.60 \\
\hline 3 & ASF-107 & 82.15 & 64.61 & 73.38 & 21.35 & 80.83 & 54.43 & 67.63 & 32.66 & 80.67 & 65.80 & 73.23 & 18.43 \\
\hline 4 & DSF-114 & 74.70 & 65.23 & 69.97 & 12.67 & 72.57 & 50.93 & 61.75 & 29.81 & 73.83 & 59.80 & 66.82 & 19.01 \\
\hline 5 & SH-177 & 91.52 & 69.93 & 80.72 & 23.58 & 86.83 & 49.50 & 68.17 & 42.99 & 90.03 & 78.23 & 84.13 & 13.11 \\
\hline 6 & DSF-104 & 72.20 & 61.73 & 66.97 & 14.50 & 75.83 & 63.10 & 69.47 & 16.79 & 88.50 & 70.23 & 79.37 & 20.64 \\
\hline 7 & RSF-106 & 81.30 & 69.93 & 75.62 & 13.98 & 79.07 & 66.13 & 72.60 & 16.36 & 75.53 & 71.23 & 73.38 & 5.69 \\
\hline 8 & DSF-111 & 83.00 & 71.40 & 77.20 & 13.98 & 79.80 & 67.53 & 73.67 & 15.37 & 80.53 & 73.03 & 76.78 & 9.31 \\
\hline 9 & RSF-107 & 78.07 & 66.20 & 72.14 & 15.20 & 83.40 & 54.80 & 69.10 & 34.29 & 85.37 & 60.80 & 73.08 & 28.78 \\
\hline 10 & ASF-104 & 79.85 & 71.20 & 75.53 & 10.83 & 79.67 & 54.00 & 66.83 & 32.22 & 80.37 & 58.60 & 69.48 & 27.08 \\
\hline 11 & TSF-106 & 80.85 & 76.32 & 78.59 & 5.60 & 79.87 & 64.50 & 72.18 & 19.24 & 79.23 & 70.73 & 74.98 & 10.73 \\
\hline \multirow[t]{2}{*}{12} & SH-491 & 88.55 & 62.33 & 75.44 & 29.61 & 90.50 & 58.07 & 74.28 & 35.84 & 91.40 & 65.17 & 78.28 & 28.70 \\
\hline & Mean & 80.90 & 68.78 & 74.84 & 14.98 & 80.54 & 59.31 & 69.93 & 26.36 & 82.38 & 68.28 & 75.33 & 17.11 \\
\hline \multicolumn{2}{|c|}{$\begin{array}{l}\mathrm{CD} \text { at } 5 \% \text { for } \\
\text { treatments }\end{array}$} & & & 1.17 & & & & 0.84 & & & & 0.78 & \\
\hline \multicolumn{2}{|c|}{$\begin{array}{l}\text { CD at } 5 \% \text { for } \\
\text { genotypes }\end{array}$} & & & 2.88 & & & & 2.05 & & & & 1.92 & \\
\hline \multicolumn{2}{|c|}{$\begin{array}{c}\text { CD at } 5 \% \text { for } \\
\text { T x G }\end{array}$} & & & 4.07 & & & & 2.9 & & & & 2.72 & \\
\hline
\end{tabular}


Table. 2 Mean of photosynthetic rate $\left(\mu \mathrm{mol} \mathrm{m} \mathrm{m}^{-2}\right)$ of sunflower cultivars during stress and after stress as influenced by moisture stress

\begin{tabular}{|c|c|c|c|c|c|c|c|c|c|c|c|c|c|}
\hline \multirow[b]{2}{*}{ S.No. } & \multicolumn{5}{|c|}{ Five days after imposition of stress } & \multicolumn{4}{|c|}{$\begin{array}{c}\text { Twenty days after imposition of } \\
\text { stress }\end{array}$} & \multicolumn{4}{|c|}{ Fifteen days after release of stress } \\
\hline & Genotype & Control & Stress & Mean & $\begin{array}{c}\% \\
\text { decrease }\end{array}$ & Control & Stress & Mean & $\begin{array}{c}\% \\
\text { decrease }\end{array}$ & Control & Stress & Mean & $\begin{array}{c}\% \\
\text { decrease }\end{array}$ \\
\hline 1 & RSF-101 & 21.30 & 18.00 & 19.65 & 15.49 & 22.87 & 20.40 & 21.63 & 10.79 & 28.33 & 22.30 & 25.32 & 21.29 \\
\hline 2 & TSF-103 & 20.33 & 18.37 & 19.35 & 9.67 & 25.00 & 23.00 & 24.00 & 8.00 & 27.73 & 25.80 & 26.77 & 6.97 \\
\hline 3 & ASF-107 & 20.97 & 20.30 & 20.63 & 3.18 & 23.57 & 22.20 & 22.89 & 5.83 & 25.84 & 22.83 & 24.34 & 11.64 \\
\hline 4 & DSF-114 & 13.20 & 10.83 & 12.02 & 17.93 & 19.40 & 17.17 & 18.28 & 11.51 & 20.67 & 19.67 & 20.17 & 4.84 \\
\hline 5 & SH-177 & 19.80 & 19.33 & 19.57 & 2.36 & 26.10 & 23.94 & 25.02 & 8.28 & 27.50 & 26.13 & 26.82 & 4.97 \\
\hline 6 & DSF-104 & 17.23 & 16.41 & 16.82 & 4.76 & 22.27 & 18.80 & 20.53 & 15.57 & 23.67 & 20.33 & 22.00 & 14.08 \\
\hline 7 & RSF-106 & 18.13 & 15.33 & 16.73 & 15.44 & 25.33 & 23.53 & 24.43 & 7.11 & 26.50 & 25.43 & 25.97 & 4.03 \\
\hline 8 & DSF-111 & 16.10 & 15.30 & 15.70 & 4.97 & 26.57 & 21.00 & 23.78 & 20.95 & 30.67 & 22.57 & 26.62 & 26.41 \\
\hline 9 & RSF-107 & 17.70 & 13.27 & 15.48 & 25.05 & 21.83 & 21.27 & 21.55 & 2.60 & 24.33 & 22.00 & 23.17 & 9.59 \\
\hline 10 & ASF-104 & 16.20 & 15.10 & 15.65 & 6.79 & 25.16 & 20.00 & 22.58 & 20.51 & 26.03 & 21.87 & 23.95 & 15.98 \\
\hline 11 & TSF-106 & 19.17 & 16.07 & 17.62 & 16.14 & 19.10 & 17.93 & 18.52 & 6.11 & 20.00 & 19.00 & 19.50 & 5.00 \\
\hline \multirow[t]{2}{*}{12} & SH-491 & 19.67 & 18.37 & 19.02 & 6.61 & 23.07 & 21.10 & 22.08 & 8.53 & 24.37 & 22.00 & 23.18 & 9.71 \\
\hline & Mean & 18.32 & 16.39 & 17.35 & 10.52 & 23.36 & 20.86 & 22.11 & 10.68 & 25.47 & 22.49 & 23.98 & 11.68 \\
\hline \multicolumn{2}{|c|}{$\begin{array}{c}\mathrm{CD} \text { at } 5 \% \\
\text { for treatments }\end{array}$} & & & 0.23 & & & & 0.19 & & & & 0.66 & \\
\hline \multicolumn{2}{|c|}{$\begin{array}{l}\text { CD at } 5 \% \text { for } \\
\text { genotypes }\end{array}$} & & & 0.56 & & & & 0.47 & & & & 1.63 & \\
\hline \multicolumn{2}{|c|}{$\begin{array}{l}\mathrm{CD} \text { at } 5 \% \text { for } \\
\mathrm{T} \times \mathrm{G}\end{array}$} & & & 0.79 & & & & 0.67 & & & & 2.3 & \\
\hline
\end{tabular}


Table.3 Mean of initial fluorescence (Fo) of sunflower cultivars during stress and after stress as influenced by moisture stress

\begin{tabular}{|c|c|c|c|c|c|c|c|c|c|c|c|c|c|}
\hline \multirow[b]{2}{*}{ S.No. } & \multicolumn{5}{|c|}{ Five days after imposition of stress } & \multicolumn{4}{|c|}{$\begin{array}{c}\text { Twenty days after imposition of } \\
\text { stress }\end{array}$} & \multicolumn{4}{|c|}{ Fifteen days after release of stress } \\
\hline & Genotype & Control & Stress & Mean & $\begin{array}{c}\% \\
\text { decrease }\end{array}$ & Control & Stress & Mean & $\begin{array}{c}\% \\
\text { decrease }\end{array}$ & Control & Stress & Mean & $\begin{array}{c}\% \\
\text { decrease }\end{array}$ \\
\hline 1 & RSF-101 & 86.67 & 84.67 & 85.67 & 2.31 & 76.33 & 76.00 & 76.17 & 0.44 & 72.33 & 69.67 & 71.00 & 3.69 \\
\hline 2 & TSF-103 & 99.33 & 92.17 & 95.75 & 7.21 & 90.33 & 82.00 & 86.17 & 9.23 & 79.67 & 70.67 & 75.17 & 11.30 \\
\hline 3 & ASF-107 & 120.00 & 90.67 & 105.33 & 24.44 & 84.00 & 83.33 & 83.67 & 0.79 & 79.00 & 66.67 & 72.83 & 15.61 \\
\hline 4 & DSF-114 & 83.33 & 80.67 & 82.00 & 3.20 & 81.00 & 78.67 & 79.83 & 2.88 & 77.00 & 73.53 & 75.27 & 4.50 \\
\hline 5 & SH-177 & 92.67 & 87.17 & 89.92 & 5.94 & 84.33 & 81.33 & 82.83 & 3.56 & 79.33 & 73.83 & 76.58 & 6.93 \\
\hline 6 & DSF-104 & 104.50 & 100.50 & 102.50 & 3.83 & 90.87 & 87.33 & 89.10 & 3.89 & 88.00 & 85.67 & 86.83 & 2.65 \\
\hline 7 & RSF-106 & 88.20 & 85.33 & 86.77 & 3.25 & 84.67 & 82.00 & 83.33 & 3.15 & 79.67 & 68.33 & 74.00 & 14.23 \\
\hline 8 & DSF-111 & 95.17 & 91.00 & 93.08 & 4.38 & 88.20 & 87.00 & 87.60 & 1.36 & 83.00 & 81.67 & 82.33 & 1.61 \\
\hline 9 & RSF-107 & 95.17 & 84.53 & 89.85 & 11.17 & 94.83 & 77.67 & 86.25 & 18.10 & 76.67 & 64.67 & 70.67 & 15.65 \\
\hline 10 & ASF-104 & 97.33 & 92.67 & 95.00 & 4.79 & 84.00 & 80.33 & 82.17 & 4.37 & 80.33 & 77.67 & 79.00 & 3.32 \\
\hline 11 & TSF-106 & 94.00 & 87.00 & 90.50 & 7.45 & 93.67 & 80.33 & 87.00 & 14.23 & 86.33 & 74.00 & 80.17 & 14.29 \\
\hline \multirow[t]{2}{*}{12} & SH-491 & 97.33 & 95.17 & 96.25 & 2.23 & 93.00 & 88.33 & 90.67 & 5.02 & 83.67 & 79.67 & 81.67 & 4.78 \\
\hline & Mean & 96.14 & 89.29 & 92.72 & 7.12 & 87.10 & 82.03 & 84.57 & 5.83 & 80.42 & 73.84 & 77.13 & 8.18 \\
\hline \multicolumn{2}{|c|}{$\begin{array}{c}\mathrm{CD} \text { at } 5 \% \\
\text { for treatments }\end{array}$} & 0.87 & & & & 0.34 & & & & 0.49 & & & \\
\hline \multicolumn{2}{|c|}{$\begin{array}{c}\mathrm{CD} \text { at } 5 \% \\
\text { for genotypes }\end{array}$} & 2.07 & & & & 0.84 & & & & 1.21 & & & \\
\hline \multicolumn{2}{|c|}{$\begin{array}{l}\text { CD at } 5 \% \text { for } \\
\text { Tx } \mathrm{G}\end{array}$} & 2.93 & & & & 1.18 & & & & 1.71 & & & \\
\hline
\end{tabular}


Table.4 Mean of maximum fluorescence (Fm) of sunflower cultivars during stress and after stress as influenced by moisture stress

\begin{tabular}{|c|c|c|c|c|c|c|c|c|c|c|c|c|c|}
\hline \multirow[b]{2}{*}{ S.No. } & \multicolumn{5}{|c|}{ Five days after imposition of stress } & \multicolumn{4}{|c|}{$\begin{array}{c}\text { Twenty days after imposition of } \\
\text { stress }\end{array}$} & \multicolumn{4}{|c|}{ Fifteen days after release of stress } \\
\hline & Genotype & Control & Stress & Mean & $\begin{array}{c}\% \\
\text { decrease }\end{array}$ & Control & Stress & Mean & $\begin{array}{c}\% \\
\text { decrease }\end{array}$ & Control & Stress & Mean & $\begin{array}{c}\% \\
\text { decrease }\end{array}$ \\
\hline 1 & RSF-101 & 237.33 & 220.00 & 228.67 & 7.30 & 208.33 & 194.33 & 201.33 & 6.72 & 192.83 & 148.00 & 170.42 & 23.25 \\
\hline 2 & TSF-103 & 313.00 & 277.83 & 295.42 & 11.24 & 246.33 & 220.67 & 233.50 & 10.42 & 211.00 & 187.00 & 199.00 & 11.37 \\
\hline 3 & ASF-107 & 307.00 & 281.67 & 294.33 & 8.25 & 297.33 & 237.67 & 267.50 & 20.07 & 266.67 & 160.00 & 213.33 & 40.00 \\
\hline 4 & DSF-114 & 295.33 & 244.67 & 270.00 & 17.16 & 276.00 & 233.67 & 254.83 & 15.34 & 232.00 & 221.33 & 226.67 & 4.60 \\
\hline 5 & SH-177 & 321.00 & 283.00 & 302.00 & 11.84 & 280.00 & 225.67 & 252.83 & 19.40 & 242.00 & 219.00 & 230.50 & 9.50 \\
\hline 6 & DSF-104 & 373.67 & 251.67 & 312.67 & 32.65 & 258.00 & 195.00 & 226.50 & 24.42 & 233.33 & 193.00 & 213.17 & 17.29 \\
\hline 7 & RSF-106 & 296.67 & 283.67 & 290.17 & 4.38 & 253.00 & 213.67 & 233.33 & 15.55 & 210.33 & 195.83 & 203.08 & 6.89 \\
\hline 8 & DSF-111 & 318.00 & 252.33 & 285.17 & 20.65 & 271.00 & 241.33 & 256.17 & 10.95 & 189.87 & 186.83 & 188.35 & 1.60 \\
\hline 9 & RSF-107 & 294.67 & 264.67 & 279.67 & 10.18 & 217.67 & 211.67 & 214.67 & 2.76 & 205.33 & 125.00 & 165.17 & 39.12 \\
\hline 10 & ASF-104 & 283.00 & 266.00 & 274.50 & 6.01 & 235.00 & 208.00 & 221.50 & 11.49 & 218.33 & 186.00 & 202.17 & 14.81 \\
\hline 11 & TSF-106 & 265.33 & 206.00 & 235.67 & 22.36 & 241.67 & 197.33 & 219.50 & 18.34 & 222.33 & 161.33 & 191.83 & 27.44 \\
\hline \multirow[t]{2}{*}{12} & SH-491 & 347.67 & 218.33 & 283.00 & 37.20 & 222.00 & 211.67 & 216.83 & 4.65 & 196.67 & 181.67 & 189.17 & 7.63 \\
\hline & Mean & 304.39 & 254.15 & 279.27 & 16.50 & 250.53 & 215.89 & \begin{tabular}{|l|}
233.21 \\
\end{tabular} & 13.83 & 218.39 & 180.42 & 199.40 & 17.39 \\
\hline \multicolumn{3}{|c|}{$\mathrm{CD}$ at $5 \%$ for treatments } & & 5.16 & & & & 1.49 & & & & 1.79 & \\
\hline \multicolumn{3}{|c|}{ CD at $5 \%$ for genotypes } & & 12.65 & & & & 3.66 & & & & 4.39 & \\
\hline \multicolumn{3}{|c|}{$\mathrm{CD}$ at $5 \%$ for $\mathrm{T} \times \mathrm{G}$} & & 17.88 & & & & 5.17 & & & & 6.21 & \\
\hline
\end{tabular}


Table.5 Mean of Maximum quantum efficiency of PS -II Photo chemistry (Fv/Fm) of sunflower cultivars during stress and after stress as influenced by moisture stress

\begin{tabular}{|c|c|c|c|c|c|c|c|c|c|c|c|c|c|}
\hline \multirow[b]{2}{*}{ S.No. } & \multicolumn{5}{|c|}{ Five days after imposition of stress } & \multicolumn{4}{|c|}{$\begin{array}{c}\text { Twenty days after imposition of } \\
\text { stress }\end{array}$} & \multicolumn{4}{|c|}{ Fifteen days after release of stress } \\
\hline & Genotype & Control & Stress & Mean & $\begin{array}{c}\% \\
\text { decrease }\end{array}$ & Control & Stress & Mean & $\begin{array}{c}\% \\
\text { decrease }\end{array}$ & Control & Stress & Mean & $\begin{array}{c}\% \\
\text { decrease }\end{array}$ \\
\hline 1 & RSF-101 & 0.74 & 0.70 & 0.72 & 6.28 & 0.68 & 0.53 & 0.61 & 22.06 & 0.62 & 0.50 & 0.56 & 19.35 \\
\hline 2 & TSF-103 & 0.72 & 0.66 & 0.69 & 8.33 & 0.71 & 0.61 & 0.66 & 14.08 & 0.63 & 0.55 & 0.59 & 12.70 \\
\hline 3 & ASF-107 & 0.70 & 0.65 & 0.68 & 8.23 & 0.68 & 0.61 & 0.65 & 10.29 & 0.65 & 0.58 & 0.61 & 10.63 \\
\hline 4 & DSF-114 & 0.73 & 0.70 & 0.71 & 4.57 & 0.68 & 0.67 & 0.68 & 1.47 & 0.64 & 0.62 & 0.63 & 2.60 \\
\hline 5 & SH-177 & 0.73 & 0.72 & 0.73 & 1.82 & 0.73 & 0.64 & 0.69 & 12.33 & 0.64 & 0.60 & 0.62 & 6.25 \\
\hline 6 & DSF-104 & 0.75 & 0.63 & 0.69 & 15.44 & 0.67 & 0.60 & 0.64 & 10.85 & 0.57 & 0.50 & 0.54 & 12.28 \\
\hline 7 & RSF-106 & 0.72 & 0.68 & 0.70 & 6.45 & 0.69 & 0.64 & 0.67 & 7.25 & 0.60 & 0.57 & 0.59 & 5.00 \\
\hline 8 & DSF-111 & 0.71 & 0.67 & 0.69 & 6.10 & 0.60 & 0.56 & 0.58 & 7.13 & 0.52 & 0.49 & 0.51 & 5.77 \\
\hline 9 & RSF-107 & 0.67 & 0.65 & 0.66 & 3.23 & 0.65 & 0.59 & 0.62 & 9.23 & 0.49 & 0.35 & 0.42 & 28.57 \\
\hline 10 & ASF-104 & 0.69 & 0.67 & 0.68 & 3.85 & 0.63 & 0.56 & 0.60 & 11.11 & 0.60 & 0.56 & 0.58 & 6.67 \\
\hline 11 & TSF-106 & 0.67 & 0.66 & 0.67 & 0.50 & 0.66 & 0.60 & 0.63 & 9.09 & 0.56 & 0.49 & 0.53 & 12.50 \\
\hline \multirow[t]{2}{*}{12} & SH-491 & 0.58 & 0.53 & 0.56 & 9.14 & 0.56 & 0.55 & 0.56 & 1.79 & 0.55 & 0.54 & 0.55 & 1.82 \\
\hline & Mean & 0.70 & 0.66 & 0.68 & 6.19 & 0.66 & 0.60 & 0.63 & 9.89 & 0.59 & 0.53 & 0.56 & 10.12 \\
\hline \multicolumn{2}{|c|}{$\begin{array}{c}\mathrm{CD} \text { at } 5 \% \\
\text { for treatments }\end{array}$} & 0.004 & & & & 0.001 & & & & 0.002 & & & \\
\hline \multicolumn{2}{|c|}{$\begin{array}{l}\mathrm{CD} \text { at } 5 \% \\
\text { for genotypes }\end{array}$} & 0.01 & & & & 0.002 & & & & 0.005 & & & \\
\hline \multicolumn{2}{|c|}{$\begin{array}{l}\mathrm{CD} \text { at } 5 \% \text { for } \\
\mathrm{T} \times \mathrm{G}\end{array}$} & 0.014 & & & & 0.003 & & & & 0.007 & & & \\
\hline
\end{tabular}


Table.6 Mean of membrane leakage $\left(\mu \mathrm{A} / \mathrm{cm}^{2}\right)$ of sunflower cultivars during stress and after stress influenced by moisture stress

\begin{tabular}{|c|c|c|c|c|c|c|c|c|c|c|c|c|c|}
\hline \multirow[b]{2}{*}{ S.No. } & \multicolumn{5}{|c|}{ Five days after imposition of stress } & \multicolumn{4}{|c|}{$\begin{array}{c}\text { Twenty days after imposition of } \\
\text { stress }\end{array}$} & \multicolumn{4}{|c|}{ Fifteen days after release of stress } \\
\hline & Genotype & Control & Stress & Mean & $\begin{array}{c}\% \\
\text { decrease }\end{array}$ & Control & Stress & Mean & $\begin{array}{c}\% \\
\text { decrease }\end{array}$ & Control & Stress & Mean & $\begin{array}{c}\% \\
\text { decrease }\end{array}$ \\
\hline 1 & RSF-101 & 7.31 & 8.37 & 7.84 & -14.40 & 10.97 & 13.50 & 12.23 & -23.10 & 13.27 & 16.00 & 14.63 & -20.60 \\
\hline 2 & TSF-103 & 5.92 & 7.51 & 6.71 & -26.87 & 10.03 & 12.96 & 11.50 & -29.21 & 12.80 & 14.60 & 13.70 & -14.06 \\
\hline 3 & ASF-107 & 7.58 & 9.77 & 8.68 & -28.79 & 11.54 & 15.07 & 13.31 & -30.52 & 15.53 & 16.27 & 15.90 & -4.72 \\
\hline 4 & DSF-114 & 6.20 & 7.50 & 6.85 & -21.03 & 10.67 & 13.93 & 12.30 & -30.63 & 15.97 & 17.97 & 16.97 & -12.53 \\
\hline 5 & SH-177 & 6.42 & 10.63 & 8.53 & -65.71 & 8.83 & 11.07 & 9.95 & -25.28 & 11.23 & 12.37 & 11.80 & -10.09 \\
\hline 6 & DSF-104 & 5.60 & 6.87 & 6.23 & -22.62 & 9.20 & 9.87 & 9.53 & -7.25 & 11.73 & 12.80 & 12.27 & -9.09 \\
\hline 7 & RSF-106 & 6.02 & 7.49 & 6.76 & -24.41 & 9.43 & 10.99 & 10.21 & -16.58 & 11.97 & 13.07 & 12.52 & -9.19 \\
\hline 8 & DSF-111 & 7.41 & 8.87 & 8.14 & -19.71 & 11.80 & 13.27 & 12.53 & -12.43 & 16.93 & 19.67 & 18.30 & -16.14 \\
\hline 9 & RSF-107 & 7.26 & 9.48 & 8.37 & -30.62 & 11.70 & 13.60 & 12.65 & -16.27 & 16.77 & 19.63 & 18.20 & -17.10 \\
\hline 10 & ASF-104 & 3.90 & 6.90 & 5.40 & -76.92 & 10.37 & 13.97 & 12.17 & -34.73 & 16.03 & 19.03 & 17.53 & -18.71 \\
\hline 11 & TSF-106 & 2.90 & 4.72 & 3.81 & -62.76 & 4.87 & 8.03 & 6.45 & -65.07 & 9.03 & 10.07 & 9.55 & -11.44 \\
\hline \multirow[t]{2}{*}{12} & SH-491 & 2.34 & 6.97 & 4.65 & -197.72 & 6.03 & 8.83 & 7.43 & -46.41 & 9.47 & 11.03 & 10.25 & -16.55 \\
\hline & Mean & 5.74 & 7.92 & 6.83 & -38.07 & 9.62 & 12.09 & 10.86 & -25.69 & 13.39 & 15.21 & 14.30 & -13.54 \\
\hline \multicolumn{2}{|c|}{$\begin{array}{l}\mathrm{CD} \text { at } 5 \% \\
\text { for treatments }\end{array}$} & & & 0.06 & & & & 0.067 & & & & 0.07 & \\
\hline \multicolumn{2}{|c|}{$\begin{array}{c}\text { CD at 5\% } \\
\text { for genotypes }\end{array}$} & & & 0.14 & & & & 0.164 & & & & 0.18 & \\
\hline \multicolumn{2}{|c|}{$\mathrm{CD}$ at $5 \%$ for $\mathrm{T} \times \mathrm{G}$} & & & 0.2 & & & & 0.231 & & & & 0.26 & \\
\hline
\end{tabular}


Table.7 Mean of SPAD meter readings and initial fluorescence (Fo) of sunflower cultivars during stress and after stress as influenced by moisture stress

\begin{tabular}{|c|c|c|c|c|c|c|c|c|c|c|c|c|c|}
\hline & \multicolumn{5}{|c|}{ Five days after imposition of stress } & \multicolumn{4}{|c|}{ Twenty days after imposition of stress } & \multicolumn{4}{|c|}{ Fifteen days after release of stress } \\
\hline S.No. & Genotype & Control & Stress & Mean & $\begin{array}{c}\% \\
\text { decrease }\end{array}$ & Control & Stress & Mean & $\begin{array}{c}\% \\
\text { decrease }\end{array}$ & Control & Stress & Mean & $\begin{array}{c}\% \\
\text { decrease }\end{array}$ \\
\hline 1 & RSF-101 & 39.67 & 32.37 & 36.02 & 18.40 & 44.93 & 33.30 & 39.12 & 25.89 & 37.83 & 31.90 & 34.87 & 15.68 \\
\hline 2 & TSF-103 & 40.00 & 39.17 & 39.58 & 2.08 & 42.53 & 35.70 & 39.12 & 16.07 & 33.67 & 30.67 & 32.17 & 8.91 \\
\hline 3 & ASF-107 & 41.47 & 40.10 & 40.78 & 3.30 & 40.4 & 39.00 & 39.70 & 1.24 & 32.33 & 31.60 & 31.97 & 2.27 \\
\hline 4 & DSF-114 & 39.87 & 39.83 & 39.85 & 0.08 & 40.23 & 37.30 & 38.77 & 7.29 & 39.60 & 35.93 & 37.77 & 9.26 \\
\hline 5 & SH-177 & 45.07 & 36.00 & 40.53 & 20.12 & 41 & 39.00 & 40.00 & 4.88 & 38.97 & 37.50 & 38.23 & 3.76 \\
\hline 6 & DSF-104 & 40.80 & 36.33 & 38.57 & 10.96 & 38.85 & 34.00 & 36.43 & 12.48 & 35.10 & 32.10 & 33.60 & 8.55 \\
\hline 7 & RSF-106 & 42.50 & 36.37 & 39.43 & 14.43 & 38.5 & 35.33 & 36.92 & 8.23 & 34.57 & 33.00 & 33.78 & 4.53 \\
\hline 8 & DSF-111 & 38.43 & 26.60 & 32.52 & 30.79 & 36.35 & 35.20 & 35.78 & 3.16 & 33.33 & 29.20 & 31.27 & 12.40 \\
\hline 9 & RSF-107 & 39.47 & 39.43 & 39.45 & 0.08 & 38.15 & 36.40 & 37.28 & 4.59 & 33.87 & 29.53 & 31.70 & 12.80 \\
\hline 10 & ASF-104 & 40.37 & 36.80 & 38.58 & 8.84 & 38.00 & 35.80 & 36.90 & 5.79 & 32.50 & 31.57 & 32.03 & 2.87 \\
\hline 11 & TSF-106 & 36.67 & 36.50 & 36.58 & 0.45 & 35.23 & 35.00 & 35.12 & 0.66 & 34.50 & 30.10 & 32.30 & 12.75 \\
\hline \multirow[t]{2}{*}{12} & SH-491 & 44.60 & 36.97 & 40.78 & 17.12 & 42.00 & 39.85 & 40.93 & 5.12 & 36.63 & 32.73 & 34.68 & 10.65 \\
\hline & Mean & 40.74 & 36.83 & 38.79 & 9.59 & 39.68 & 36.78 & 38.23 & 7.30 & 35.24 & 32.15 & 33.70 & 8.76 \\
\hline \multicolumn{3}{|c|}{$\mathrm{CD}$ at $5 \%$ for treatments } & & 0.31 & & & & 0.28 & & & & 0.32 & \\
\hline \multicolumn{3}{|c|}{ CD at $5 \%$ for genotypes } & & 0.75 & & & & 0.68 & & & & 0.78 & \\
\hline \multicolumn{3}{|c|}{$\mathrm{CD}$ at $5 \%$ for $\mathrm{T} \times \mathrm{G}$} & & 1.06 & & & & 0.96 & & & & 1.11 & \\
\hline
\end{tabular}


Table.8 Mean of specific leaf area $\left(\mathrm{cm}^{2} \mathrm{~g}^{-1}\right)$ of sunflower cultivars during stress and after stress as influenced by moisture stress

\begin{tabular}{|c|c|c|c|c|c|c|c|c|c|c|c|c|c|}
\hline \multirow[b]{2}{*}{ S.No. } & \multicolumn{5}{|c|}{ Five days after imposition of stress } & \multicolumn{4}{|c|}{$\begin{array}{c}\text { Twenty days after imposition of } \\
\text { stress }\end{array}$} & \multicolumn{4}{|c|}{ Fifteen days after release of stress } \\
\hline & Genotype & Control & Stress & Mean & $\begin{array}{c}\% \\
\text { decrease }\end{array}$ & Control & Stress & Mean & $\begin{array}{c}\% \\
\text { decrease }\end{array}$ & Control & Stress & Mean & $\begin{array}{c}\% \\
\text { decrease }\end{array}$ \\
\hline 1 & RSF-101 & 90.67 & 75.33 & 83.00 & 16.91 & 157.33 & 97.67 & 127.50 & 37.92 & 177.00 & 116.33 & 146.67 & 34.27 \\
\hline 2 & TSF-103 & 109.33 & 82.67 & 96.00 & 24.39 & 142.67 & 102.00 & 122.33 & 28.50 & 153.33 & 127.67 & 140.50 & 16.74 \\
\hline 3 & ASF-107 & 115.00 & 99.00 & 107.00 & 13.91 & 188.00 & 142.67 & 165.33 & 24.11 & 273.00 & 232.00 & 252.50 & 15.02 \\
\hline 4 & DSF-114 & 97.33 & 85.00 & 91.17 & 12.67 & 221.00 & 153.33 & 187.17 & 30.62 & 280.33 & 230.67 & 255.50 & 17.72 \\
\hline 5 & SH-177 & 86.33 & 71.67 & 79.00 & 16.99 & 98.33 & 87.00 & 92.67 & 11.53 & 106.00 & 97.00 & 101.50 & 8.49 \\
\hline 6 & DSF-104 & 132.00 & 100.00 & 116.00 & 24.24 & 154.67 & 108.00 & 131.33 & 30.17 & 169.80 & 111.67 & 140.73 & 34.24 \\
\hline 7 & RSF-106 & 100.00 & 80.33 & 90.17 & 19.67 & 136.33 & 122.33 & 129.33 & 10.27 & 163.00 & 133.33 & 148.17 & 18.20 \\
\hline 8 & DSF-111 & 118.67 & 70.97 & 94.82 & 40.20 & 155.00 & 108.67 & 131.83 & 29.89 & 189.37 & 140.33 & 164.85 & 25.89 \\
\hline 9 & RSF-107 & 118.67 & 89.00 & 103.83 & 25.00 & 136.67 & 117.67 & 127.17 & 13.90 & 164.67 & 134.00 & 149.33 & 18.62 \\
\hline 10 & ASF-104 & 101.00 & 70.67 & 85.83 & 30.03 & 107.67 & 96.67 & 102.17 & 10.22 & 116.67 & 105.00 & 110.83 & 10.00 \\
\hline 11 & TSF-106 & 133.00 & 85.33 & 109.17 & 35.84 & 195.00 & 145.00 & 170.00 & 25.64 & 286.67 & 167.33 & 227.00 & 41.63 \\
\hline \multirow[t]{2}{*}{12} & SH-491 & 97.33 & 61.67 & 79.50 & 36.64 & 98.33 & 73.33 & 85.83 & 25.42 & 110.67 & 72.00 & 91.33 & 34.94 \\
\hline & Mean & 108.28 & 80.97 & 94.62 & 25.22 & 149.25 & 112.86 & 131.06 & 24.38 & 182.54 & 138.94 & 160.74 & 23.88 \\
\hline \multicolumn{2}{|c|}{$\begin{array}{l}\mathrm{CD} \text { at } 5 \% \\
\text { for treatments }\end{array}$} & & & 0.86 & & & & 0.77 & & & & 0.93 & \\
\hline \multicolumn{2}{|c|}{$\begin{array}{c}\mathrm{CD} \text { at } 5 \% \\
\text { for genotypes }\end{array}$} & & & 2.12 & & & & 1.89 & & & & 2.28 & \\
\hline \multicolumn{2}{|c|}{$\begin{array}{l}\mathrm{CD} \text { at } 5 \% \\
\text { for } \mathrm{T} \times \mathrm{G}\end{array}$} & & & 2.99 & & & & 2.68 & & & & 3.23 & \\
\hline
\end{tabular}


Environmental stresses that affect PSII efficiency lead to a characteristic decrease in the Fv/Fm ratio (Reddy et al., 2004). Fv/Fm decreased with increase in age of the crop. The reduction in Fv/Fm was maximum at 15 days after release of stress $(10.12 \%)$ than that of at 20 days after imposition of stress $(9.89 \%)$ compared to their controls (Table 5). Significant difference in Fv/Fm was seen in cultivars. Genotypes exhibited significant variation in $\mathrm{Fv} / \mathrm{Fm}$. At 15 days after stress release, ASF-107 under control and DSF-114 under stress and interaction recorded higher Fv/Fm and were superior over other genotypes. Any decrease in optimal quantum yield directly decreases the flux of electrons out of PSII and consequently lowers the rates of ATP and NADPH2 formation and that, in turn, slows the enzymatic conversion of $\mathrm{CO}_{2}$ into organic carbon (Schofield et al., 1995). Membrane leakage increased with increase in stress duration. Membrane leakage increased in stress treatments compared to control (Table 6). At 15 days after release of stress membrane leakage increased under stress compared to control. Among genotypes TSF106 exhibited lower membrane leakage both under control and stress as well as in interaction. In interaction, ASF-107 recorded minimum reduction in membrane leakage among cultivars and maximum reduction in membrane leakage was recorded in RSF-101. When plants are under high-temperature induced water stress, the structure of membranes is altered, permeability increases, electrolyte leakage increases, and eventually the cell dies (Wang, 1988).

The present investigation, SPAD chlorophyll meter reading of 12 genotypes was significantly affected by stress. It was decreased with increase in stress duration. SPAD meter reading was (38.79) at 5 days after imposition of stress at it reduced to (38.23) at 20 days after imposition of stress (Table 7). SPAD chlorophyll reading showed a declining trend with approaching the end of the plant growth period showing normal pattern of leaf senescence. During stress release period, among the genotypes DSF-114 followed by $\mathrm{SH}-177$ under control and $\mathrm{SH}-$ 177 under stress exhibited higher SPAD chlorophyll meter reading in comparison to other genotypes. Whereas in mean effect, $\mathrm{SH}$ 177 (38.23) and DSF-114 (37.77) maintained higher SPAD values and were superior over other genotypes. SPAD chlorophyll meter reading (SCMR), a reflection of leaf chlorophyll/ leaf nitrogen declined in stress treatment due to degradation of leaf chlorophyll content.

Specific leaf area (SLA), an indication of leaf thickness found to be significantly different in plants of control and stress treatments at 3 growth stages. Under control condition, SLA was high compared to stress condition (Table 8). At 75 DAS, genotype SH-491 recorded lowest specific area in control, stress and genotype $\mathrm{x}$ treatments interaction, whereas DSF-111 recorded significantly superior specific leaf area over other genotypes in interaction at 75 DAS. Reduction of SLA under stress is due to reduction in leaf area without concomitant reduction in leaf thickness. In fact, reduction in leaf area under stress is serving as one of the strategies to survive under stress. Nageswara Rao and Wright (1994) reported that, in groundnut, genotypes with lower SLA (thicker leaves) had more photosynthetic machinery and the potential for greater assimilation per unit leaf area.

Based on results obtained it can be concluded that water stress induced at flower bud initiation stage reduced water status of plant tissue (RWC) alters membrane permeability causing solute leakage. Degradation of chlorophyll molecule, reduction in photosynthetic rate and photo system II (FV/FM) is consequence of membrane 
damage. Lower SLA under water stress is adaptation mechanism to survive the drought. Genotypes SH-177, SH-491 and DSF-111 are considered to be promising line as they showed better performance then remaining genotype grown under drought for RWC, photosynthetic rate, leaf fluorescence, membrane integrity, SPAD and SLA. These lines may be studied with molecular tools extensively for future harvest enhancement by incorporating drought tolerant gene incorporation.

\section{References}

Jha, B.N. and Singh, R.A. 1997. Physiological responses of rice varieties to different levels of moisture stress. Indian J. Plant Physiol., 2: 81-84.

Lawlor, D.W. 2002. Limitation to photosynthesis in water stress; stomata Vs metabolism and the role of ATP: Annl. Bot., 89: 871-885.

Miyashita, K., Tanakamaru, S., Maitani, T. and Kimura, K. 2004. Recovery responses of photosynthesis, transpiration, and stomatal conductance in kidney bean following drought stress. Environ. Exp. Botany, In press.

Reddy, A.R., Chaitanya, K.V. and Vivekanandan, M. 2004. Droughtinduced responses of photosynthesis and antioxidant metabolism in higher plants. J. Plant Physiol., 161: 11891202.

Sinclair, T.R. and Ludlow, M.M. 1985. Who taught plants thermodynamics? The unfulfilled potential of plant water potential. Australian J. Plant Physiol., 12: 213-217.

Umashaanker, R. 1991. Gametophytic screening techniques in identification and development of drought tolerant lines. Proceedings of National Symposium. Recent Advances. Drought Research, Dec, 10-13, kottayam, Kerala India, pp.5.

\section{How to cite this article:}

Geetha, A., A. Sivasankar, P. Saidaiah and Lakshmi Prayaga. 2017. Effect of Moisture Stress on Key Physiological Parameters in Sunflower Genotypes. Int.J.Curr.Microbiol.App.Sci. 6(5): 147-159. doi: http://dx.doi.org/10.20546/ijcmas.2017.605.018 